\title{
Short-Term Effects of COVID-19-Related Deferral of Intravitreal Injection Visits
}

This article was published in the following Dove Press journal:

Clinical Ophthalmology

\section{Noy Ashkenazy (D) \\ Lediana Goduni \\ William E Smiddy}

Department of Ophthalmology, Bascom Palmer Eye Institute, University of Miami Miller School of Medicine, Miami, FL, USA
Correspondence: William E Smiddy Tel + I 305-326-6I72

Email wsmiddy@med.miami.edu
Purpose: To determine secondary effects of the mandated COVID-19 pandemic closure period for elective treatment on non-elective, injection-based retina care and outcomes.

Patients and Methods: In this cross-sectional, retrospective analysis of a single-provider outpatient clinic across multiple satellites, consecutive patients returning for intravitreal injections (IVIs) of anti-vascular endothelial growth factor or corticosteroids were identified as "delayed" or "undelayed" during a six-week study interval during the COVID-19 pandemic that closely following a mandated period of prohibited elective encounters. A "delayed" encounter was defined as having a follow-up interval exceeding $33 \%$ of the recommended cycle. Patients seen for IVIs during the corresponding six-week interval a year previously were identified for study as pre-COVID-19 controls. Main outcome measures included bestcorrected visual acuity (BCVA) and optical coherence tomography (OCT) assessment based on findings of intraretinal or subretinal fluid consistent with new or recurrent neovascular events. Results: The study included 183 eyes of 144 patients who underwent IVI-based care from June 18, 2020, through August 7, 2020, compared to 193 eyes of 154 patients injected during the corresponding interval 1 year before. There were 62 eyes of 46 patients seen in the study period later than scheduled (among 144 patients of 183 eyes in total), which represented a larger proportion of delayed patients compared to the previous year $(31.9 \%$ vs. $14.9 \%$, $\mathrm{p}<0.0005)$. Considering the patterns from the control group, the attributed delay due to COVID-19 was $15.0 \%$ of patients. The delayed return eyes had a greater decline in BCVA (3 letters), higher rates of worsened OCT results $(48 \%)$, and prompted reduction in previously prescribed injection intervals $(\mathrm{p}<0.02)$.

Conclusion: The unintended consequence of delayed care of patients on established care regimens should be anticipated, and mitigate strategies considered if similar restrictions are mandated in the future.

Keywords: COVID-19, SARS-CoV-2, pandemic, retinal vascular diseases, anti-vascular endothelial growth factor, treatment outcomes

\section{Plain Language Summary}

The purpose of this investigation was to determine the short-term impact of COVID-19 pandemic shutdown mandates on patients returning for previously established injectionbased retinal treatment regimens.

Consecutive patients returning for intravitreal injections (IVIs) of anti-vascular endothelial growth factor or corticosteroids during a six-week study interval during the COVID-19 pandemic that closely following a mandated period of prohibited elective encounters. This included IVI patients returning later than scheduled and returning as scheduled. Patients seen for IVI from the corresponding interval a year previously were identified for study as preCOVID-19 controls. 
The results of this single-provider, cross-sectional study demonstrated more frequent delayed appointments, resulting in higher rates of declined visual acuity, worsened OCT results, and reduced prescribed injection intervals, when compared to those who continued their scheduled intervals.

Many patients suffering from retinal vascular diseases were adversely impacted by a delay in their regular intravitreal injections as a result of the initial pandemic shutdown. This unintended consequence should be anticipated with attempts to mitigate if similar restrictions are mandated in the future or in considering any external disruption of care.

\section{Introduction}

COVID-19 was first identified as a public health hazard in late December $2019^{1}$ and was accorded pandemic status on March 11, 2020. ${ }^{2}$ The national task force recommended, as part of its response to mitigate spread, a temporary nationwide halt on non-urgent procedures in the United States. ${ }^{2,3}$ Consequently, an executive order by the Florida governor authorized only urgent encounters beginning on March 17, 2020 and ending on May 11, $2020 .^{4}$

The American Academy of Ophthalmology and the American Society of Retinal Specialists formulated guidelines defining urgent conditions to offer guidance. ${ }^{5,6}$ Vision-threatening conditions were authorized for visits including intravitreal injections (IVIs) for various prevalent retinal vascular conditions. ${ }^{6}$ Despite administrative efforts to maintain urgent appointments, some patients thought the clinic was completely closed or elected to defer their appointments due to personal concerns.

We studied the characteristics and results of patients undergoing IVIs who re-established care during a six-week interval shortly following the restricted period.

\section{Methods}

All studies were conducted after approval of Human Subjects Committee of the University of Miami which granted a waiver of study consent due to the low risk to the patient and the retrospective nature of the study, and in accordance with the Declarations of Helsinki. We reviewed a six-week study interval (from 6/18/2020 to 8/7/2020), a month after rescission which was chosen to allow for a transition to "new normal" operations. Inclusion criteria included adults ages 18 and over who had been engaged in active IVI treatment (defined as having had at least 3 prior visits) for a diagnosis of macular edema or neovascular complications. Exclusions included repeated injections within the study interval and eyes that had not been seen 3 times or within the previous 12 months.

One retina specialist saw all patients during the study interval across four practice sites using the same electronic record. Standard protocols were enacted to maximize patient safety, including universal masks, social distancing through restrictions of visitors, use of plastic barriers at desks, and compulsive hand-washing. The previous three visits were reviewed to identify a delayed encounter which was defined as when the delay exceeded $33 \%$ of the previously scheduled follow-up cycle (eg, returning at $>8$ weeks instead of 6 weeks). A pre-COVID-19 control group was assembled from encounters during the corresponding interval in 2019 . The treating physician typically (but not using a standard protocol) asked the patient why their visit was delayed, taking care not to compound their anxieties of missing the appointment.

Clinical information analyzed is listed in Supplemental Table 1. Outcome measures included BCVA (logMAR) change, OCT status (better, stable, worse), switch of intravitreal agent, and change in previously established followup interval. The OCT was judged to be worse when there was an increase in subretinal or intraretinal fluid; all changes were not subtle.

Statistical analyses were performed using the SPSS 26.0 (SPSS Inc, Chicago, IL) software package. Analyses were performed using Pearson $\chi^{2}$ analysis, Fisher exact test, and $t$-test, when appropriate. A p-value of 0.05 or lower was considered statistically significant.

\section{Results}

The number of IVIs in the 2020 study interval was $9.9 \%$ (219 vs. 243) lower than for the corresponding 2019 preCOVID-19 control group (Table 1). After exclusions, 183 eyes of 144 patients were identified during the study interval (study group), and 193 eyes of 154 patients during the corresponding pre-COVID-19 interval (control group).

The study group included $62 / 183(33.9 \%)$ eyes of $46 /$ $144(31.9 \%)$ patients whose visit was delayed and 121/183 (66.1\%) eyes of $98 / 144(68.1 \%)$ patients whose visit was not delayed. In contrast, in the 2019 control group 26/193 (13.5\%) eyes of $23 / 154(14.9 \%)$ patients had been delayed and $167 / 193(86.5 \%)$ eyes of $131 / 154(85.1 \%)$ patients were not delayed (both $\mathrm{p}<0.0005$ ). Thus, the number of delayed visits attributable to the COVID-19 closure was $20.4 \%$ of eyes and $15.0 \%$ of patients.

The mean delay in the study group was 3.08 (SD 5.70) weeks, compared to 1.50 (SD 7.29) weeks in the 2019 
Table I Detailed Counts of Included and Excluded Intravitreal Injections (IVI) and Study Patients of the 2019 and 2020 Cohorts

\begin{tabular}{|c|l|l|}
\hline Study Period & Patients & Eyes \\
\hline 2020 Total & 175 & 219 \\
Excluded & 31 & 36 \\
Second encounter & 16 & 20 \\
$>12$ months since last visit & 6 & 6 \\
$<3$ injection visits & 9 & 10 \\
Study Group 2020 & 144 & 183 \\
Delayed & 46 & 62 \\
Not Delayed & 98 & 121 \\
\hline 2019 Total & 200 & 243 \\
Excluded & 46 & 50 \\
Second encounter & 16 & 19 \\
$>12$ months since last visit & 17 & 17 \\
$<3$ injection visits & 13 & 14 \\
Control Group 2019 & 154 & 193 \\
Delayed & 23 & 26 \\
Not Delayed & 131 & 167 \\
\hline
\end{tabular}

cohort $(\mathrm{p}=0.021)$. Delayed encounters in the study group were attributable to patient concern for COVID-19 in 20/ $46(43.5 \%)$ patients, other personal reasons in $10(6.6 \%)$ patients, unspecified in 15/46 (32.6\%) patients, and illness during the closure period in $1 / 46(2.2 \%)$. One patient each in the delayed and not delayed group indicated having had COVID-19 infection.

The delayed subgroup had a larger proportion with at least a 0.05 logMAR (3 letters) loss in BCVA, poorer OCT results $(50 \%)$, and more often was switched to different IVI agents compared to those not delayed $(p<0.03)$ (Table 2). There were no statistically significant factors across the subgroups associated with worsening at re-presentation. However, eyes with wAMD were less frequently delayed compared to pseudophakic cystoid macular edema $(5 / 6$, $83.3 \%)$ and diabetic macular edema (21/45, 46.7\%) $(\mathrm{p}=0.009)$ (Supplemental Table 1).

\section{Discussion}

This retrospective study showed $32 \%$ of patients returning for established IVIs were delayed in their follow-up during a six-week study interval shortly following the restricted interval; half of the delays were attributable to COVID-19 concerns. The magnitude of the disruption might be even larger, since nearly $10 \%$ fewer incident IVI patients were seen compared to a corresponding control group. Moreover, the consequences of delay included worse BCVA loss and more frequent worsening in the OCT features. This is one of the first studies to show worse visual acuities and OCT findings in eyes whose IVIs were delayed due the COVID-19 pandemic and is unique in that it surveyed results of those re-presenting rather than just those not presenting.

Delayed IVI care is not unique to the constraints of the pandemic closure and has been shown by others to be associated with inferior outcomes in retinal vascular diseases. ${ }^{7-9}$ Relatedly, we acknowledge that alterations in the management plan (changing agents, shortening of subsequent follow-up intervals) were subjective, based on the recommendations and potential biases of the treating physician.

Obeid et al studied 9007 wet AMD patients in which 22\% were lost to follow up for over 12 months after IVI. Risk factors for failure to return included lack of VA improvement, female gender, non-Caucasian, distance residing from the office, and unilateral versus bilateral treatment. ${ }^{10}$ Gao et al reported $25 \%$ of 3400 patients with retinal vein occlusion with macular edema were lost to follow-up over 12 months. Risk factors included non-Caucasian, $<65$ years, and poorer baseline vision. ${ }^{11}$ The current study did not demonstrate these risk factors, but was substantially smaller and was conducted under a different setting.

Lange et al estimated that by May 2020, nearly $41 \%$ of adults had delayed medical care due to fear of the pandemic, corresponding to marked reductions in both routine and emergency encounters for life-threatening conditions. ${ }^{12}$ Recent studies show that the 2020 pandemic closure resulted in a 53-75\% reduction in IVIs when compared to $2019 .^{4,13,14}$ Our study demonstrates a relatively lower, but significant, $10 \%$ overall decrease in IVI volume in 2020 after the severest of restrictions were rescinded compared to the same period in 2019, with over 2.5 times as many delayed intravitreal injection treatment encounters. At least $42 \%$ of these delayed IVIs were directly related to pandemic-related constraints.

Reports from other countries have suggested a similar experience as is related in the current manuscript. Saleh et al reported that intravitreal injections were safely delivered, without any know Covid-19 case transmission in a tertiary care clinic in Jordan, but clinical volumes were diminished due to patient fears regarding contracting the virus. ${ }^{15}$ Moreover, those who did seek care had poorer presenting visual acuity. Two studies from Milan, Italy also documented decreased clinical (up to $71 \%$ ) and injection (54-58\%) volumes. ${ }^{13,16}$ There was a higher rate of submacular hemorrhage among wAMD patient reported ${ }^{16}$ 
Table 2 Outcome Measures for Delayed and Not Delayed Groups

\begin{tabular}{|c|c|c|c|}
\hline & Delayed n (\%N), N=62 & Not Delayed $n(\% N), N=|2|$ & p-value \\
\hline Visual Acuity Change (AlogMAR) & - & - & $0.006 *$ \\
\hline$\leq 0.05$ (Snellen equivalent, $<3$ letter loss, or gain) & $30(48.4)$ & $84(69.4)$ & - \\
\hline$>0.05$ (Snellen equivalent, >3 letter loss) & $32(51.6)$ & $37(30.6)$ & \\
\hline Visual Acuity (Snellen) at Study Visit & - & - & $0.322 *$ \\
\hline$\geq 20 / 40$ & $15(24.2)$ & $42(34.7)$ & - \\
\hline $20 / 50-20 / 200$ & $38(61.3)$ & $66(54.5)$ & - \\
\hline$<20 / 200$ & $9(14.5)$ & $13(10.7)$ & - \\
\hline Optical Coherence Tomography Status & - & - & $<0.001 *$ \\
\hline Better & $6(9.7)$ & $31(25.6)$ & - \\
\hline Stable & $26(38.8)$ & $78(64.5)$ & - \\
\hline Worse & $30(48.4)$ & $12(9.9)$ & - \\
\hline Change in Intravitreal Agent & $4(6.5)$ & $2(1.7)$ & $0.084 *$ \\
\hline Recommended Follow-Up Interval, Mean (SD) & - & - & - \\
\hline Difference in follow-up interval (weeks) & $-1.94(18.07)$ & $-0.08(2.86)$ & $0.011^{\mathrm{a}}$ \\
\hline
\end{tabular}

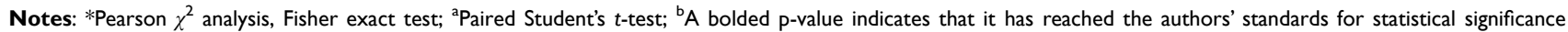
$(p<0.05)$.

Abbreviations: SD, standard deviation.

and a higher rate of absence among elderly and wAMD compared to other diagnostic categories. ${ }^{13}$

Diabetics and the elderly wARMD represent groups that are at higher risk of complications from COVID-19 infection. Understandably, those patients frequently chose not to risk coming to their appointments. A dependence on travel arrangements to appointments, the need to quarantine for those in assisted living facilities, inaccurate information regarding their healthcare appointments, and illness were also likely was also additional factors in reduced compliance with IVI-based follow up during the COVID-19 pandemic period.

The current study captured cases that re-presented after a delay, rather than ascertaining the cohort that did not present as previously scheduled. The retrospective study design did not permit us to collect the reason for the delayed return in a standardized fashion.

In conclusion, the potentially deleterious effects of the pandemic closure period require careful consideration if future healthcare constraints are imposed. Clear messaging to define and to distinguish urgent and non-urgent categories of medical disease is essential. The degree of recovery after reestablishing care merits further investigation.

\section{Author Contributions}

All authors made a significant contribution to the work reported, whether that is in the conception, study design, execution, acquisition of data, analysis and interpretation, or in all these areas; took part in drafting, revising or critically reviewing the article; gave final approval of the version to be published; have agreed on the journal to which the article has been submitted; and agree to be accountable for all aspects of the work.

\section{Funding}

Supported by NIH Center Core Grant P30EY014801, Research to Prevent Blindness Unrestricted Grant.

\section{Disclosure}

Dr Noy Ashkenazy served in the Advisory Board for Alimera Sciences in May 2020. The authors report no other potential conflicts of interest (financial and/or non-financial) for this work.

\section{References}

1. Zhu N, Zhang D, Wang W, et al. A novel coronavirus from patients with pneumonia in China, 2019. NEJM. 2020;382(8):727-733. doi:10.1056/NEJMoa2001017

2. WHO. Director-General's Opening Remarks at the Media Briefing on COVID-19-11 March 2020 [Press Release]. Who.it. World Health OrganizationMarch; 2020.

3. Khan M, Sivalingam A, Haller JA. Perceptions of occupational risk and changes in practice of U.S. vitreoretinal surgery fellows during the COVID-19 pandemic. Ophthalmol Retina. 2020;22:S24686530(2420)30203-30207.

4. Wasser L, Weill Y, Brosh K. et al. The impact of COVID-19 on intravitreal injection compliance. SN Compr Clin Med. 2020:1-4. doi:10.1007/s42399-020-00614-4 
5. ASRS. ASRS Releases Guidelines to Help Retina Practices Navigate COVID-19 Pandemic. American Society of Retina Specialists; March, 2020. Available from: https://www.asrs.org/clinical/clinicalupdates/1962/asrs-releases-guidelines-to-help-retina-practicesnavigate-covid-19-pandemic. Accessed January 19, 2021.

6. AAO. List of urgent and emergent ophthalmic procedures. March, 2020. Available from: https:/www.aao.org/headline/listof-urgent-emergent-ophthalmic-procedures. Accessed January 19 , 2021 .

7. Holekamp NM, Campbell J, Almony A, et al. Vision outcomes following anti-vascular endothelial growth factor treatment of diabetic macular edema in clinical practice. Am J Ophthalmol. 2018;191:83-91. doi:10.1016/j.ajo.2018.04.010

8. Soares RR, Mellen P, Garrigan H, et al. Outcomes of eyes lost to follow-up with neovascular age-related macular degeneration receiving intravitreal anti-vascular endothelial growth factor. Ophthalmol Retina. 2020;4:134-140. doi:10.1016/j.oret.2019.07.010

9. Hsu J, Matsunaga D, Obeid A, Wibbelsman TD, Wu C. Outcomes of eyes with diabetic macular edema that are lost to follow-up after anti-vascular endothelial growth factor therapy. Paper presented at Retina Society 2020 VR, the 53rd annual scienti; 2020.

10. Obeid A, Gao X, Ali FS, et al. Loss to follow-up among patients with neovascular age-related macular degeneration who received intravitreal anti-vascular endothelial growth factor injections. JAMA Ophthalmol. 2018;136(11):1251-1259. doi:10.1001/jamaophthalmol.2018.3578

11. Gao X, Obeid A, Adam MK, et al. Loss to follow-up in patients with retinal vein occlusion undergoing intravitreal anti-VEGF injections. Ophthalmic Surg Lasers Imaging Retina. 2019;50(3):159-166. doi:10.3928/23258160-20190301-05
12. Lange S, Ritchey MD, Goodman AB, et al. Potential indirect effects of the COVID-19 pandemic on use of emergency departments for acute life-threatening conditions - United States, January-May 2020. MMWR Morb Mortal Wkly Rep. 2020;69(25):795-800. doi:10.15585/ mmwr.mm6925e2

13. Borrelli E, Grosso D, Vella G, et al. Impact of COVID-19 on outpatient visits and intravitreal treatments in a referral retina unit: let's be ready for a plausible "rebound effect". Graefes Arch Clin Exp Ophthalmol. 2020;258(12):2655-2660.

14. Carnevali A, Giannaccare G, Gatti V, et al. Intravitreal injections during COVID-19 outbreak: real-world experience from an Italian tertiary referral center. Eur J Ophthalmol. 2020;1-3.

15. Saleh OA, Jammal H, Alqudah N, Alqudah A, Abu-Yaghi N. Clinical experience in the administration of intravitreal injection therapy at a Tertiary University Hospital in Jordan during the COVID-19 lockdown. Clin Ophthalmol. 2020;14:2473-2480. doi:10.2147/ OPTH.S269179.

16. Romano F, Monteduro D, Airaldi M, et al. Increased number of submacular hemorrhages as a consequence of coronavirus disease 2019 lockdown. Ophthalmol Retina. 2020;4(12):1209-1210. doi:10.1016/j.oret.2020.06.027.
Clinical Ophthalmology

\section{Publish your work in this journal}

Clinical Ophthalmology is an international, peer-reviewed journal covering all subspecialties within ophthalmology. Key topics include: Optometry; Visual science; Pharmacology and drug therapy in eye diseases; Basic Sciences; Primary and Secondary eye care; Patient Safety and Quality of Care Improvements. This journal is indexed on PubMed
Dovepress

Central and CAS, and is the official journal of The Society of Clinical Ophthalmology (SCO). The manuscript management system is completely online and includes a very quick and fair peer-review system, which is all easy to use. Visit http://www.dovepress.com/ testimonials.php to read real quotes from published authors. 